\title{
Memory transfer in meal-worms
}

PETER O. PERETTI and HAROLD G. WAKELEY, Illinois institute of Technology, Chicago, Ill. 60616

Some studies have been done with memory transfer in lower organisms with the planaria. In research of this type, the animals of one group are usually trained to specific stimuli, killed, and then fed to other animals of the same species on which testing for memory transfer will occur. The following research consists of an investigation of memory transfer within the same organism as it itself passes, through metamorphosis, from one form to another. It was found that memory transfer does occur and might be explained as a result of internal rather than external factors.

Walker (1966) noted the conditioning rates of r'anarians which had ingested light-shock conditioned worms, as well as planarians which had ingested shockexposed, light-exposed, and light-shock extinguished animals. The former organisms were the experimental group; the latter were the controls.

In the results he shows that none of the cannibals in the experimental group demonstrated a systematic increase in the CR over 75 trials. These results were used to support the hypothesis that tissue sensitivity rather than memory transfer had occurred.

In a follow-up study, Walker \& Milton (1966) performed a somewhat similar study with planarians to support an analogous hypothesis. Their results were in agreement with those from the above investigation. Again, it was assumed that tissue sensitivity rather than memory transfer had occurred.

Corning \& John (1961) had planarians perform a simple learning task and then cut them in two parts. One-half of the animals heads and tails were placed in Ribonuclease. an enzyme which breaks down RNA, while the other half were placed in ordinary pond water. Those heads and tails which regenerated in ordinary pond water did show evidence of retention over the performance of those animals which had regenerated in the cnzyme. The researchers suggested the importance of RNA in memory transfer.

Byme, Samuel, Bennett, Rosenzweig, \& Wasserman (1906) have studied the importance of RNA in memory transfer in rats. In their study, they injected $\mathrm{RN} A$ from the brains of trained rats into a naive group and then tested the performance of the untrained animals.

\section{MEAI . WORM STAGI Subjects}

Twenty meal-nurms (Tonchrio moliter) were randomly divided into two groups.
There were 10 animals in the experimental group, and 10 organisms in the control.

\section{Apparatus}

The apparatus developed was a Y maze which was useful in running the worms as well as the beetles. The three arms were separately constructed of interleaved coils of No. 16 tinned bus wire.

Both coils for a single arm were wound simultaneously around a suitable rod and evenly spaced at the completion of the coiling process. Spacing between coils was about from $1 / 32$ to $1 / 16$ in. Coils were placed as close together as possible in order not to permit the worm or beetle to escape the apparatus at points other than the starting opening and goal or end points. Spaces between the coils were necessary, however, to possibly probe or prod the worm in the maze when it might continually not respond to the shock.

The completed arms were glued to a styrene plastic base with styrene cement. Leads were allowed to protrude from each arm and could be connected to the shock supply via clips and toggle switches.

In its application, shock was obtained from a pair of bell transformers with the secondaries connected to provide isolation from ground. A $25 \mathrm{k}$ ohm $2 . \mathrm{W}$ poten tiometer connected across the output transformer permitted current adjustment. This instrument was sufficiently versatile to permit shock to the animals, at any position in each maze arm, as each segment was separate from the others.

Procedure
Preliminary procedures included the orientation of the 20 meal-worms to the maze; running of each of the animals in both arms of the maze six times to avoid a preference effect, and determination of a shocking procedure which would motivate the animals toward the "correct" arm while inhibiting their entrance to the "incorrect" arm.

Criteria for the learning performance were that the experimental meal-worms, given the same number of trials as the control group. must turn to the left-hand arm on three consecutive trials with a mean number of errors less than 10 per trial.

Animals from the experimental group were shocked whenever they tried to enter the "incorrect" right-hand arm of the maze. The shock was applied contirinously until they turned to the left and entered the "correct" left hand arm. No such shock was given the cuntrol meal-worms. Both groups of animals would he shocked if they tried to come back down the starting arm, if if they failed to show some movemuent in the total ma/c arcia.
The meal-worms were run for 8 days, because the criteria could not be met before the 8 th day.

RESULTS AND DISCUSSION

Figure 1 shows that during the first 3 days of the experiment, the mean number of errors for the experimental and control groups was about the same (Experimental, 25; Control, 26). By the 4th day, the animals from both groups began to respond much differently. Experimental mealworms had a mean number of 14 errors, while the control organisms had a mean number of 31 errors. At this point, it was inferred that learning had begun to occur in the experimental group.

From the 5 th to the 7 th days, the experimental worms did much better than the controls in the mean errors per trial (Experimental, 11; Control, 25). The experimental animals no longer needed the electric shock for the motivation down the "correct" arm, and they rarely needed shock at the choice point.

Considering all of the trials, the worms of the experimental group did significantly better in the learning of the $Y$ maze than the controls when comparing their mean number of errors per trial. The grand mean for the experimental group was 17 , while the grand mean for the control group was 26 . Considering only the last 3 days of trials, the experimental mean was as low as 11 , while the control group mean was as high as $25.1 \mathrm{t}$ was assumed that learning had occurred for the experimental animals.

Mean number of trials for the experimental group was the same for each day; however, the mean number of errors was 29 on the first day and decreased to only seven by the 8 th day. Trials for the controls were the same for all days. Variations in mean number of errors was less for this group than for the experimental. The range of errors was from as high as 31 to a low of 24 mean errors.

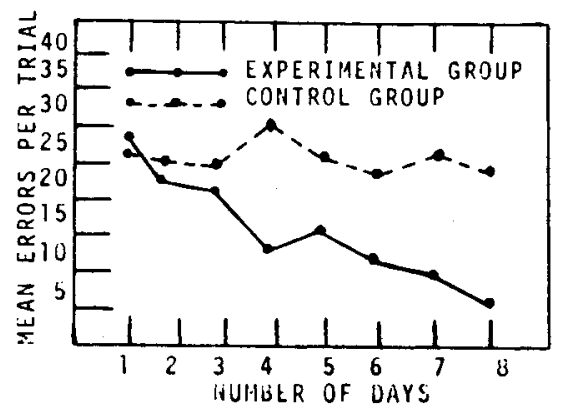

Fig. I. Comparison of Y-maze learning between the experimental and control meal-worm groups. 


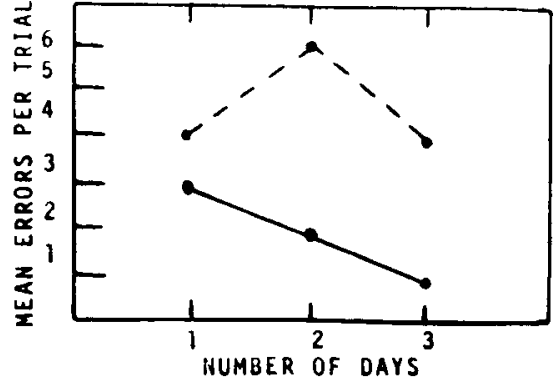

Fig. 2. Comparison of Y.maze learning between the experimental and control beetle groups.

Speed of the run was an important consideration for the meal-worm stage. For the experimental group, none of the animals were removed from the $Y$ maze for violation of a "no movement" criteria. This criteria stated that if a worm stayed in one arm and showed no movement for a consecutive 10 -min period, then it could be taken from the arm and run from the starting point another time. The longest running time from the starting arm to the end of the left-hand goal arm was 52 min during the first 5 days of trials for the slowest crawling worm, while the shortest running time was $21 \mathrm{~min}$. By the 6th day of trials, the longest run on the last three trials was $45 \mathrm{~min}$ for one worm, while the shortest was a mere $6 \mathrm{~min}$. On the 8 th day, the experimental animals were going through the entire maze correctly in $4.5 \mathrm{~min}$.

A total of 23 worms were pulled (some worms were pulled more than once) out of the maze in the control group for violation of the 10-min "no movement" criteria during the 8-day run. The longest crawling time from the starting arm to the end of the left-hand arm was 94 min during the first 5 days of trials for the slowest worm, while the shortest time was $32 \mathrm{~min}$. By the 6 th day of trials, the longest run on the last 3 days was still over an hour ( $78 \mathrm{~min}$ ) for one worm, while one animal had the shortest run for the group of $30 \mathrm{~min}$.

Errors were scored using the criteria: turning around in the maze; turning around and moving out of the starting arm; facing in the correct direction but moving backward; entering the "incorrect" right-hand arm with more than one-half the body; and attempting to crawl out of the maze coils with more than one-half of the body exposed to the outside.
BEETLESTAGI

Subjects

The same 20 meal-worms which had been used in the above experiment served as Ss. They had now gone through the stage of metamorphosis and had become dark brown beetles. Experimental worms above became the experimental beetles, while the control meal-worms were designated as the control group of beetles.

\section{Apparatus}

Instrumentation for the beetles was the same as for the meal-worms.

Procedure

Preliminary procedures again included the orientation of the 20 beetles to the $\mathrm{Y}$ maze; running of each of the animals in both arms of the maze six times to avoid a preference effect, and the determination of a shocking procedure which was found to be much shorter for these quick-moving beetles.

Criteria for learning performance were that the experimental beetles, given the same number of trials as the control group, must tum to the left-hand arm on three consecutive trials with a mean number of errors less than two per trial.

As with the worms, the experimental group of beetles was shocked whenever an animal attempted to enter the "incorrect" right-hand maze of the apparatus, while no shock was given the controls.

Criteria was met by the experimental group by the end of the 3rd day of trials. RESULTS AND DISCUSSION

Figure 2 shows that the experimental beetles showed a definite increase in learning the maze when compared with the control animals. The figure also indicates that both groups of beetles far outlearned their worm-like forms.

The mean number of errors for the experimental group of beetles on the first 2 days was 2.5 , while the mean number of errors for the control animals was 5 . All members of the experimental group did seem to have a "sense of direction" for turning left as soon as the preference trials, during the preliminary procedure, had been completed.

By the 3rd day, the animals in the experimental group were running toward the "correct" left-hand arm with less than two errors per trial. Their speed was slightly faster than the speed for members of the control group, regardless of the selection of the arm the controls might choose. Experimental animals ran the maze in from
$10 \mathrm{sec}$, for the quickest heetle, to $34 \mathrm{sec}$, for the slowest. The mean speed was $18 \mathrm{sec}$. Control animals ran the maze in from $14 \mathrm{sec}$, for the quickest time, to $52 \mathrm{sec}$ for the slowest; the mean time was $23 \mathrm{sec}$

No animals had to be pulled for not running. They were quick to move when placed in the starting arm, especially if given an initial shock at the starting point.

CONCLUSIONS

Meal-worms can learn to run a $Y$ maze. This learning does seem to transfer from the worm stage to the beetle stage. This conclusion is based on the mean number of errors for both the worm and the beetle in the experimental as compared with the control groups. Time might also be considered in the learning process, as both the experimental worms and beetles ran the maze faster than the worms and beetles of the control group.

Tissue sensitivity, extent of RNA transfer, or what have you might account for this transfer of memory from the worm form to the beetle form. Our major purpose was to suggest that such memory transfer can be observed in the meal-worm. This has been done. Hypotheses might be generated from these results and conclusions leading to future testing of them.

Because of the conditions of the external environment, which were held constant for both the worms and beetles, it was assumed that the learning which did take place was a result of the internal environment of the organisms. Physiological and biological factors, including tissue sensitivity and RNA might be useful in memory transfer. These factors are assumed to remain somewhat constant in the meal-worm when it goes through its metamorphosis stage. Although its outward structure and bodily form do change, relevant physiological and biological aspects concerned with niemory transfer seem to remain stable and enhance performance of the beetle on task s to which the worm had previously been trained.

\section{REFERENCES}

BYRNE, W. L., SAMUEL, D., BENNETT, E. L., ROSENZWEIG, M. R., \& WASSERMAN, E. Memory transfer. Science, 1966, 15 3, 658-659. CORNING, W. C., \& JOHN, F. R. Effects of ribonucleose in retention of conditioned responses in regenerated planarians. Science, 1961, 134, 1363-1365.

WALKER, D. R. Memory transfer in planarians: An artifact of experimental variables. Psychonomic Science, 1966, 5, 357-358.

WALKER, D. R., \& MILTON, G. A. Memory transfer vs sensitization in cannibal planarians. Psychonomic Science, 1966, 5, 293-294. 